\title{
ORIGINAL ARTICLE Reference values for leptin and adiponectin in children below the age of 10 based on the IDEFICS cohort
}

\author{
E Erhardt ${ }^{1,10}$, R Foraita ${ }^{2,10}$, I Pigeot ${ }^{2,3}$, G Barba ${ }^{4}$, T Veidebaum $^{5}$, M Tornaritis ${ }^{6}$, N Michels ${ }^{7}$, G Eiben ${ }^{8}$, W Ahrens ${ }^{2,3}$, LA Moreno ${ }^{9}$, \\ E Kovács ${ }^{1}$ and D Molnár ${ }^{1}$ on behalf of the IDEFICS consortium
}

OBJECTIVE: To establish age- and sex-specific reference values for serum leptin and adiponectin in normal-weight 3.0-8.9-year old European children.

SUBJECTS AND METHODS: Blood samples for hormone analysis were taken from 1338 children of the IDEFICS (Identification and prevention of Dietary- and lifestyle-induced health Effects in Children and infantS) study cohort. Only normal-weight children aged 3.0-8.9 years were included $(n=539)$ in our analysis. Using the General Additive Model for Location Scale and Shape, age- and sex-specific percentiles were derived. The influence of under/overweight and obesity on the proposed reference curves based on normal-weight children was investigated in several sensitivity analyses using the sample without obese children ( $n=1015)$ and the whole study sample $(n=1338)$.

RESULTS: There was a negative age trend of adiponectin blood levels and a positive trend of leptin levels in boys and girls. Percentiles derived for girls were generally higher than those obtained for boys. The corresponding age-specific differences of the 97th percentile ranged from -2.2 to $4.6 \mathrm{~g} \mathrm{ml}^{-1}$ and from 2.2 to $4.8 \mathrm{ng} \mathrm{ml}^{-1}$ for adiponectin and leptin, respectively.

CONCLUSIONS: According to our knowledge, these are the first reference values of leptin and adiponectin in prepubertal, normal-weight children. The presented adiponectin and leptin reference curves may allow for a more differentiated interpretation of children's hormone levels in epidemiological and clinical studies.

International Journal of Obesity (2014) 38, S32-S38; doi:10.1038/ijo.2014.133

\section{INTRODUCTION}

Adipose tissue secretes a number of biologically active adipokines, which are low-molecular weight peptides. These peptides have numerous central and peripheral functions and have an important role in fat- and whole-body metabolism. ${ }^{1}$ Leptin and adiponectin have endocrine and metabolic functions. Leptin was the first identified adipocytokine secreted mainly in white adipose tissue, but it has also been detected in many other tissues including placenta, endometrium and in gastrointestinal tracts. Leptin contributes to body weight regulation through modulating feeding as a satiety signal and stimulates energy expenditure. ${ }^{2,3}$ Serum leptin concentrations are increased in obese male and females compared with non-obese individuals. A distinct dimorphism can be found between boys and girls especially during progressive pubertal stages. Matsubara et al., ${ }^{4}$ who investigated only obese women, reported that serum leptin concentrations are increased as a consequence of 'leptin resistance'. Levels of leptin are related to body fat mass and fat distribution, and they change during puberty. ${ }^{5}$ According to recent findings, leptin may be a link between obesity and metabolic disorders. ${ }^{6}$ It is known that leptin deficiency leads to obesity, but also to diabetes and to reproductive dysfunction. Leptin also has a role in immune response; it modulates the monocytes and macrophages, natural killers and dendritic cells.
Adiponectin is one of the most abundant plasma proteins produced in white adipose tissue. Plasma adiponectin concentrations were found to be decreased in obese patients, in the case of type 2 diabetes mellitus, insulin resistance and dyslipidaemia and it is known to be a marker of the metabolic syndrome. ${ }^{6,7}$ Pubertal development can influence serum adiponectin levels, as indicated by the observed difference between sexes during early adulthood. ${ }^{2,7}$

According to a literature review, normative values of leptin and adiponectin for children are mainly based on small national study populations. $^{8-11}$ European reference standards from unselected populations are missing. Therefore, the main objective of this paper is to establish age- and sex-specific reference values for leptin and adiponectin, based on a European sample of normalweight children aged 3.0-8.9 years from eight European countries who participated in the baseline examinations of the IDEFICS (Identification and prevention of Dietary- and lifestyle-induced health Effects in Children and infantS) study.

\section{SUBJECTS AND METHODS}

Study subjects

A cohort of 16228 children aged 2-9 years was examined in a populationbased baseline survey in eight European countries ranging from North to South and from East to West (Sweden, Germany, Hungary, Italy, Cyprus,

${ }^{1}$ Department of Paediatrics, University of Pécs, Pécs, Hungary; ${ }^{2}$ Leibniz Institute for Prevention Research and Epidemiology-BIPS, Bremen, Germany; ${ }^{3}$ Institute of Statistics, Faculty of Mathematics and Computer Science, Bremen University, Bremen, Germany; ${ }^{4}$ Epidemiology and Population Genetics, Institute of Food Science, National Research Council, Avellino, Italy; ${ }^{5}$ Department of Chronic Diseases, National Institute for Health Development, Tallinn, Estonia; ${ }^{6}$ Research and Education Institute of Child Health, Strovolos, Cyprus; ${ }^{7}$ Department of Public Health, University of Ghent, Ghent, Belgium; ${ }^{8}$ Department of Public Health and Community Medicine, University of Gothenburg, Gothenburg, Sweden and ${ }^{9}$ Growth, Exercise, Nutrition and Development (GENUD) Research Group, University of Zaragoza, Zaragoza, Spain. Correspondence: Dr È Erhardt, Department of Paediatrics, University of Pécs, József A. str. 7., 7623 Pécs, Hungary.

E-mail: erhardt.eva@pte.hu

${ }^{10}$ These authors equally contributed to this work. 
Spain, Belgium, Estonia) from autumn 2007 to spring 2008. The study was not designed to provide a representative sample for each country. Rather, this baseline survey was the starting point of a prospective cohort study with the largest European children's cohort established to date. Additional 2517 new children were included in the follow-up survey (2009-2010). All children in the defined age group who resided in the defined regions and who attended the selected primary schools (grades 1 and 2), preschools or kindergartens were eligible for participation. Children were approached via schools and kindergartens to facilitate equal enrolment of all social groups. All study centres obtained ethical approval from their local Ethics Committees. In addition to the signed informed consent given by parents, each child was asked to give verbal assent immediately before examination. Participants were free to opt out of specific modules like blood drawing. The study design has been described elsewhere. ${ }^{12}$

Inclusion and exclusion criteria for the analysis group

Hormones were measured in a subsample of IDEFICS children from the baseline survey selected for a nested case-control study on overweight and obesity. All children exceeding the 85th percentile of age- and sexspecific body mass index (BMI) of the IDEFICS cohort were eligible as cases. These comprise all children classified as obese according to the IOTF criteria $^{13}$ and a sample of children in the upper range of the overweight category. Controls were selected at random from the remaining children, individually matched to cases by age ( \pm 0.5 years of age), sex and study centre. To qualify for inclusion in the case-control study, the parental questionnaires, the 24-h dietary recall and several anthropometric measurements had to be available for each child. This restriction resulted in 1328 case-control pairs providing these required measurements and fasting blood. Together with a few additional children, 1338 children aged 2.0-9.9 years provided hormone measurements from fasting blood samples. Of these, 60 children were excluded due to small numbers of children younger than 3.0 years and older than 8.9 years. Seven hundred and thirty-nine children were not classified as normal weight according to Cole and Lobstein ${ }^{14}$ and were excluded because body fat is associated with hormone levels, thus leaving 539 children for analysis (see Figure 1). The log-transformed hormone measurements contained no outliers (defined as values outside the range of mean \pm 4 s.d.).
Blood hormone sampling

Fasting venous blood was collected from all children consenting to provide biological material. Serum total leptin and adiponectin were only measured in children selected for the case-control study. Serum samples were frozen $\left(-80^{\circ} \mathrm{C}\right)$ and shipped to a central laboratory on dry ice (The Laboratoriumsmedizin Dortmund $\mathrm{Dr}$ Eberhard und Partner GbR is accredited according to DIN EN ISO 15189 by the German Accreditation Council). Concentration of serum leptin $\left(\mathrm{ng} \mathrm{ml}^{-1}\right.$ ) was measured using the MEDIAGNOST (Reutlingen, Germany) enzyme immunoassay. The measurable range for leptin was $0.5-64 \mathrm{ng} \mathrm{ml}^{-1}$. The sensitivity of the leptin assay was less than $0.1 \mathrm{ng} \mathrm{ml}^{-1}$. Adiponectin was measured by enzyme-linked immunosorbent assay using the MEDIAGNOST (Reutlingen, Germany) kit. The sensitivity of the adiponectin assay was less than $0.6 \mathrm{\mu g} \mathrm{ml}^{-1}$, whereas the measurable range was $2-100 \mu \mathrm{g} \mathrm{ml}^{-1}$.

\section{Statistical methods}

We calculated percentile curves of adiponectin and leptin as a function of age as a continuous variable, stratified by sex using the General Additive Model for Location Scale and Shape (GAMLSS). ${ }^{15}$ The GAMLSS method is an extension of the LMS method ${ }^{16}$ that models three parameters: the median (M) models the outcome variable depending on one explanatory variable, and the coefficient of variation $(S)$ accounts for the variation around the mean and adjusts for non-uniform dispersion, whereas the skewness parameter (L) accounts for the deviation from a normal distribution using a Box-Cox transformation. The GAMLSS method is able to additionally model the kurtosis using other distributions and to include more than one covariate. We used the gamlss package (version 4.2-6) ${ }^{17}$ of the statistical software R (version 3.0.1). Different distributions, that is, the Box-Cox Cole and Green, gamma or inverse Gaussian distribution were fitted to the observed distribution of adiponectin and leptin. Moreover, the influence of age on parameters of the considered distributions were modelled either as a constant, as a linear function or as a penalised spline of the covariate. Goodness of fit was assessed by the Bayesian Information Criterion, Q-Q plots and the sample percentages below each centile curve to select the final model including the fitted distribution of adiponectin and leptin and the influence of age on distribution parameters. Worm plots were used as a diagnostic tool to assess whether adjustment for kurtosis was required..$^{18}$ Finally, percentile curves for the $1 \mathrm{st}$, 3rd, 10th, 25th, 50th,

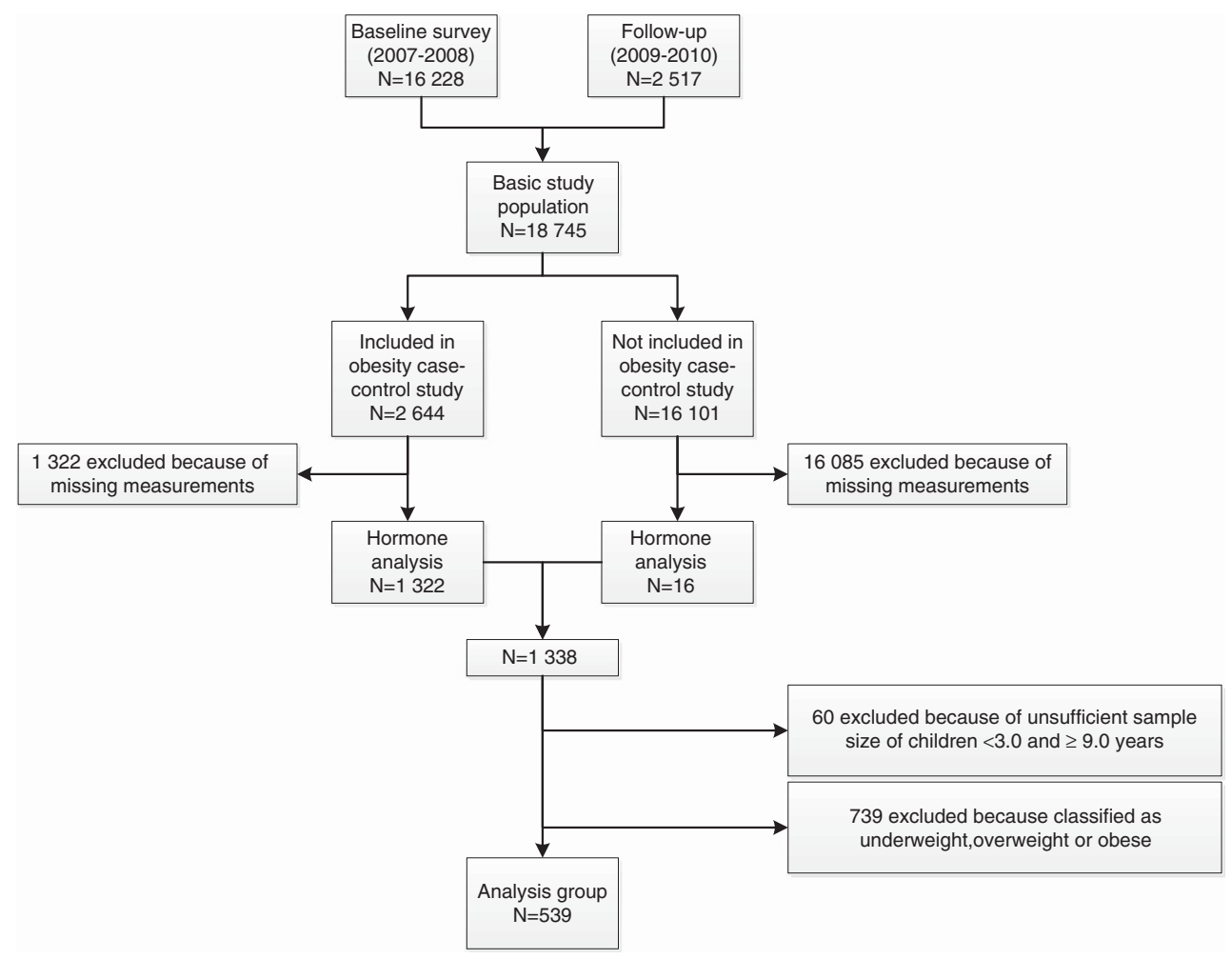

Figure 1. Flow chart showing the selection of the analysis group. 
75th, 90th, 97th and 99th percentiles were calculated based on the model that showed the best goodness of fit.

The final model for leptin is based on an inverse gamma distribution for girls, modelling $\log (\mu), \log (\sigma)$ and $v$ as constant. For boys, a Box-Cox power exponential distribution was used, modelling $\log (\mu)$ as penalised beta spline depending on age, $\log (\sigma)$ as linear function of age, and $v$ and $\tau$ as constant. With regard to adiponectin, a gamma distribution was fitted for boys and girls considering $\log (\mu)$ as linear function of age and $\log (\sigma)$ as linear function of age for girls and as constant for boys.

In further sensitivity analyses, we investigated the influence of fat on leptin and adiponectin concentrations by comparing our analysis group of normal-weight children with the whole sample of children between 2.0 and 9.9 years who provided hormone measurements $\left(n=1338, n_{\text {girls }}=687\right.$, $\left.n_{\text {boys }}=651\right)$ and with the sample only excluding the obese children ( $\left.n=1015, n_{\text {girls }}=531, n_{\text {boys }}=484\right)$ regarding the 3th, 50th and 97th percentile curves. For all of the sensitivity analyses except one, we used the same distributions as for the main analysis. The analysis of leptin in boys for the whole study sample was conducted using a generalised inverse gamma distribution, modelling $\log (\mu)$ and $v$ as a function of age and $\log (\sigma)$ as constant.

\section{RESULTS}

Basic characteristics of the study population are presented as mean values ( \pm s.d.) or median (interquartile range) by 1-year age groups in Table 1. The analysis group consists of 283 girls and 256 boys. The BMI is similar in boys and girls, but body fat mass as well as adiponectin and leptin levels generally show a trend for higher values in girls than in boys.

Percentiles of adiponectin and leptin are presented in 1-year age groups in Table 2, Figures 2 and 3, respectively. Adiponectin values are higher in girls than in boys aged 5.0 years and older. Across all age groups, the difference between girls and boys ranges from -2.2 to $4.6 \mu \mathrm{g} \mathrm{ml}^{-1}$ for the 97 th percentile of adiponectin. There is a negative age trend of the mean adiponectin concentrations in both sexes, whereas there is a positive age trend of the variance of adiponectin in girls and a negative trend in boys.

Leptin values are generally higher in girls than in boys. Across all age groups, the difference between boys and girls ranges from 2.2 to $4.8 \mathrm{ng} \mathrm{ml}^{-1}$ for the 97 th percentile of leptin. Leptin concentrations show a positive nonlinear trend with age above the 50th percentiles and a rather constant trend up to the 50th percentile. There is also a positive age trend for the variance of leptin values in both sexes.

Sensitivity analyses are presented in Figures 4 and 5 comparing percentiles for children of different BMI categories. Percentile values for adiponectin levels show minor differences for boys of the whole study sample as compared with the values for normalweight boys. The differences are more pronounced for girls: the inclusion of obese girls pushes the percentile curves downwards. The characteristics of the leptin curves change distinctly with the inclusion of under/overweight and obese children. The percentile curves are dramatically shifted upwards for the whole sample with age in both sexes. Smaller deviations from the normal-weight percentiles can already be detected for the 10th percentile for girls older than 7 years and boys older than 8 years. Leptin values are generally higher in girls than in boys for all BMI categories.

\section{DISCUSSION}

This study presents reference values of leptin and adiponectin in mostly prepubertal, normal-weight children. Although these reference percentiles are based on a small subgroup of the IDEFICS cohort, only, this is the first time that reference values of leptin and adiponectin are proposed based on a multinational non-clinical population.

We restricted our analysis group to normal-weight children as especially leptin is known to be associated with fat mass. ${ }^{5}$ In our

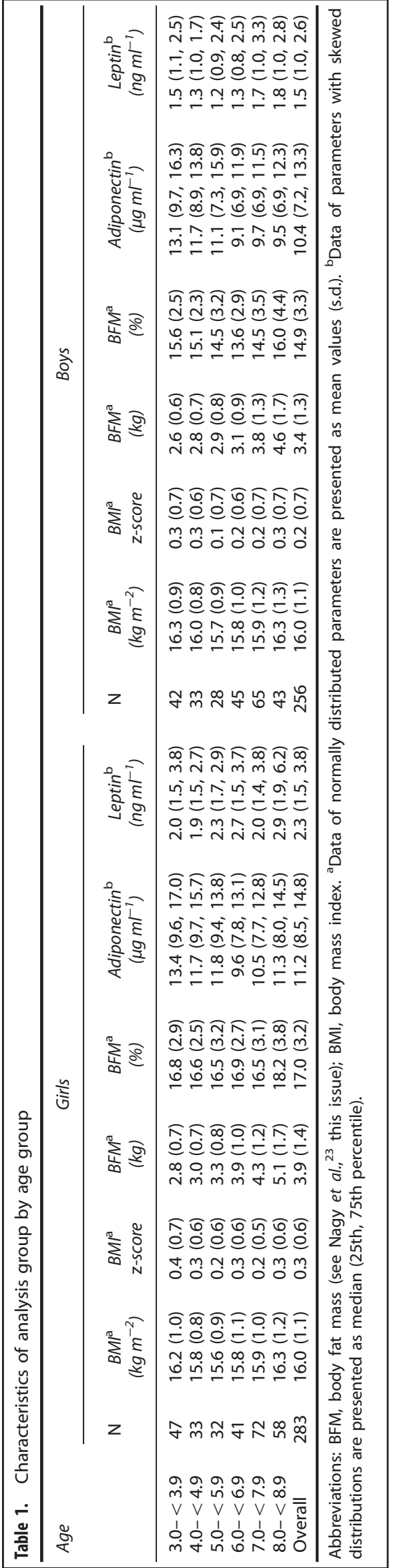


sensitivity analyses, the influence of BMI on leptin is clearly seen, especially for the upper percentiles.

In our sample, adiponectin and leptin levels were slightly higher in girls than in boys, which is in accordance with the findings in children $^{3,19}$ and in adults, ${ }^{4}$ reporting higher leptin levels in females than in males.

There are some studies in healthy children on the national level, ${ }^{8-10,20}$ but their comparability with our results is limited mostly because of either a different age distribution of the sample or due to incomparable laboratory analyses. Pre-analytical (for example, serum vs plasma) and analytical factors (laboratory method) can significantly affect measured concentrations of leptin and adiponectin. Concerning leptin, the first main problem is that laboratory methods may not be comparable. Enzyme-linked immunosorbent assay demonstrates a wider leptin range than radio immunoassay. ${ }^{9,16}$ Plasma concentrations of hormones are generally higher than serum concentrations, partly due to the removal of fibrinogens in the serum. ${ }^{21}$ In addition, not only total, but also either free or bound leptin concentrations have been reported. L'Allemand et al., ${ }^{10}$ for instance, specifically measured free leptin. In a Greek study, ${ }^{9}$ the enzyme-linked immunosorbent assay method was used to examine the serum leptin concentration of 294 children (154 boys). The mean age ( \pm s.d.) of the children was $9.3( \pm 1.8)$ years, and thus the average age is 3 years higher than in our study. Another Greek study ${ }^{8}$ reported total leptin levels of healthy, non-obese children, but it cannot be compared with our results, because plasma leptin levels were measured and the mean age of the children was higher than in our cohort (mean age \pm s.d.: $10.5 \pm 1.5$ vs $6.2 \pm 1.7$ years).

A paper by Venner et al. ${ }^{11}$ reviewed studies reporting leptin reference ranges in prepubertal children. Their conclusion was that only limited articles investigating leptin levels in children are available in contrast to the numerous publications in human adults. Their opinion was that one should be cautious referring to published leptin values and means because of the dependency of leptin levels on age and $\operatorname{sex}^{8,22}$ and the pre-analytical and analytical sources of variation. As it is very difficult to interpret individual leptin values, there is a need to establish reference values for leptin concentrations in prepubertal children.

With respect to adiponectin, most previous studies cannot be compared with our results due to different age ranges of children in these studies and the IDEFICS sample, considering the fact that adiponectin levels change with age. There is only one Mexican cross-sectional study ${ }^{19}$ that investigated 190 school-age children classified as obese and 196 children classified as eutrophic. A significant difference $(P<0.001)$ was found between eutrophic and obese children with respect to anthropometric and metabolic measurements. The mean adiponectin concentration in the Mexican study was $15.5 \mu \mathrm{g} \mathrm{ml}^{-1}$ in normal-weight children (mean age \pm s.d.: $9.4 \pm 1.8$ years), whereas in our cohort, that is on average, 3 years younger, it was $11.4 \mathrm{\mu g} \mathrm{ml}^{-1}$ (mean age \pm s.d.: $6.2 \pm 1.7$ years). Difference in adiponectin concentrations between the two studies further supports the age dependency of adiponectin.

According to our results, adiponectin percentiles show a negative age trend and leptin levels above the 50th percentile generally show a positive trend with age in both sexes. It is known that obese individuals have lower adiponectin and higher leptin levels compared with normal-weight individuals. ${ }^{20}$ The leptin levels reflect changes in body fat content as the main site of leptin production is the white adipose tissue. Changes in the investigated hormone levels over age in the IDEFICS sample may reflect the age-related changes in body composition (see Nagy et al., ${ }^{23}$ this issue), that is, the increase in percent body fat. The development of the hormone levels over age were reported by others previously. Murphy et al. ${ }^{20}$ showed an age-dependant increase of leptin and a decrease of adiponectin in British children between the ages of 5 and 8 years. A Spanish study ${ }^{22}$ examining 

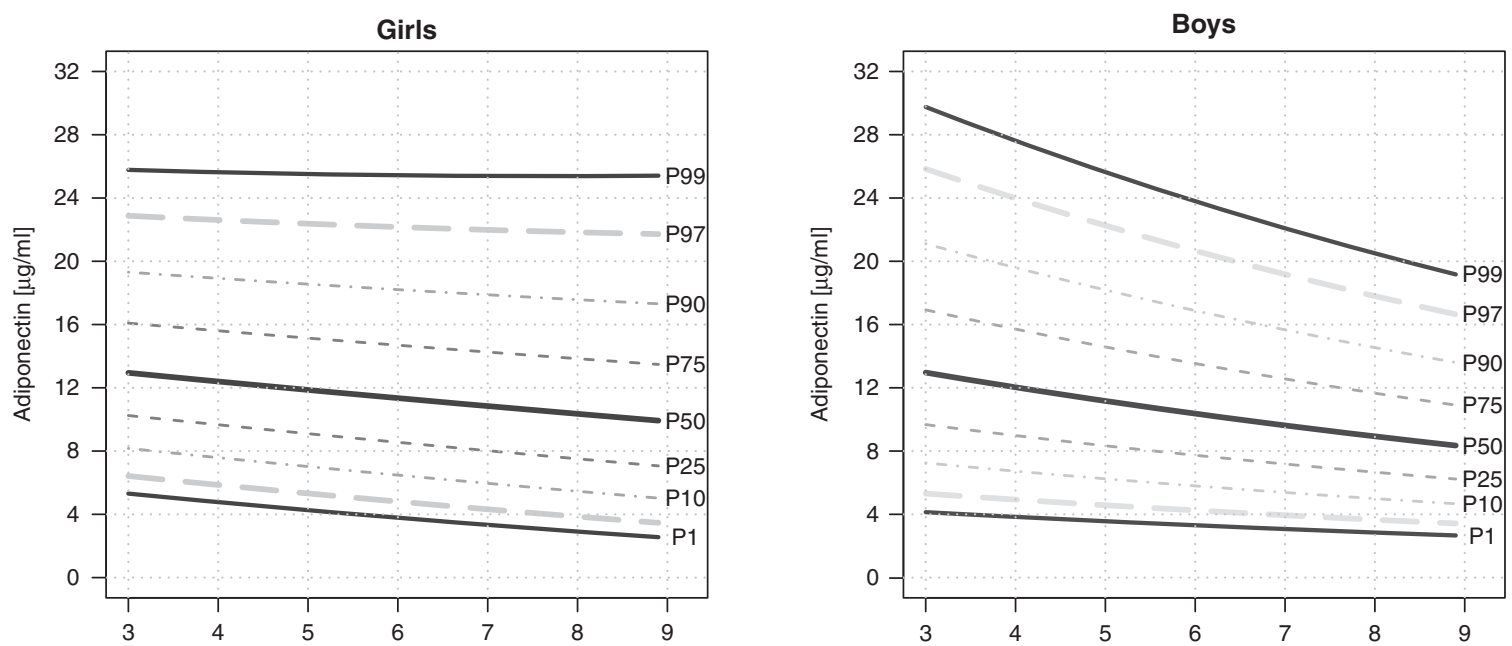

Figure 2. Smoothed percentile curves for adiponectin $\left(\mu \mathrm{g} \mathrm{ml}^{-1}\right)$ in girls and boys by age among normal-weight children aged 3.0-8.9 years modelled by GAMLSS.
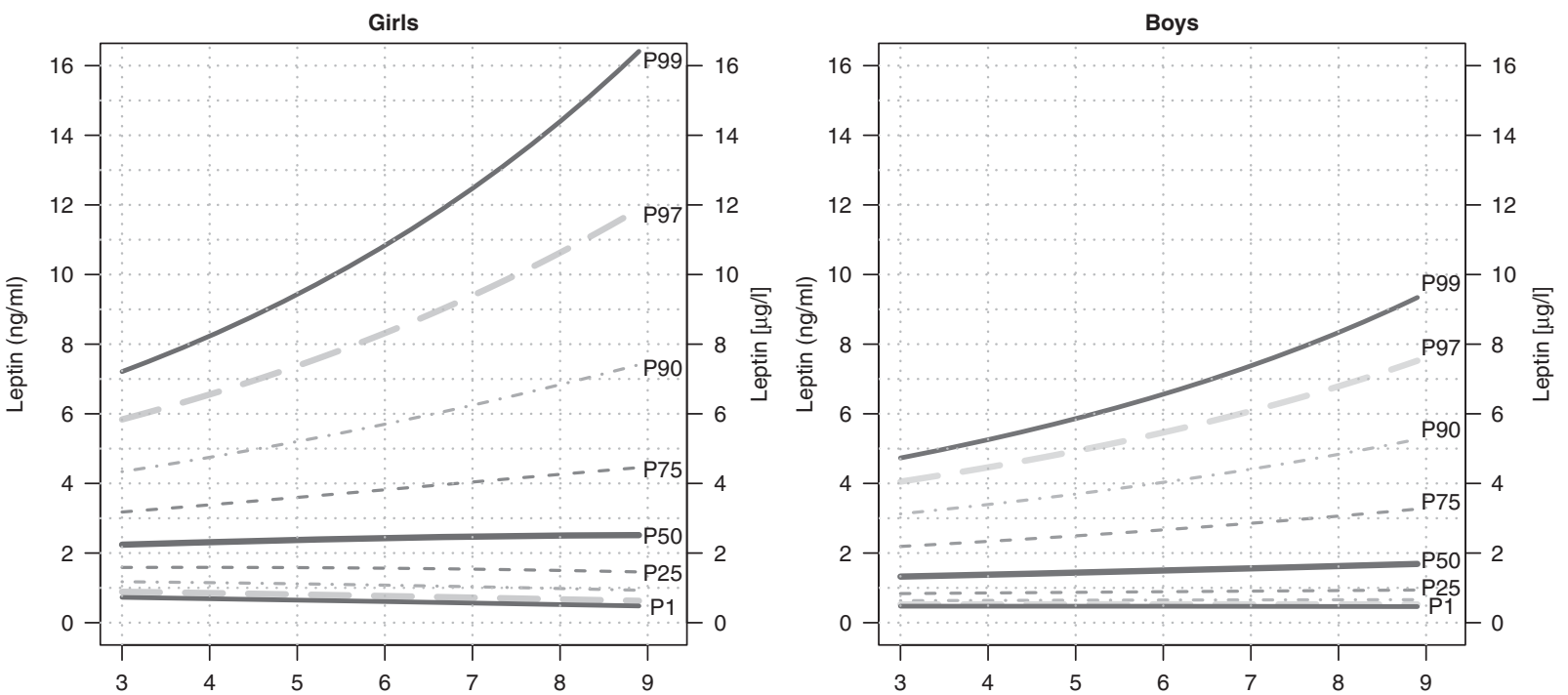

Figure 3. Smoothed percentile curves for leptin $\left(\mathrm{ng} \mathrm{ml}^{-1}\right)$ in girls and boys by age among normal-weight children aged 3.0-8.9 years modelled by GAMLSS.
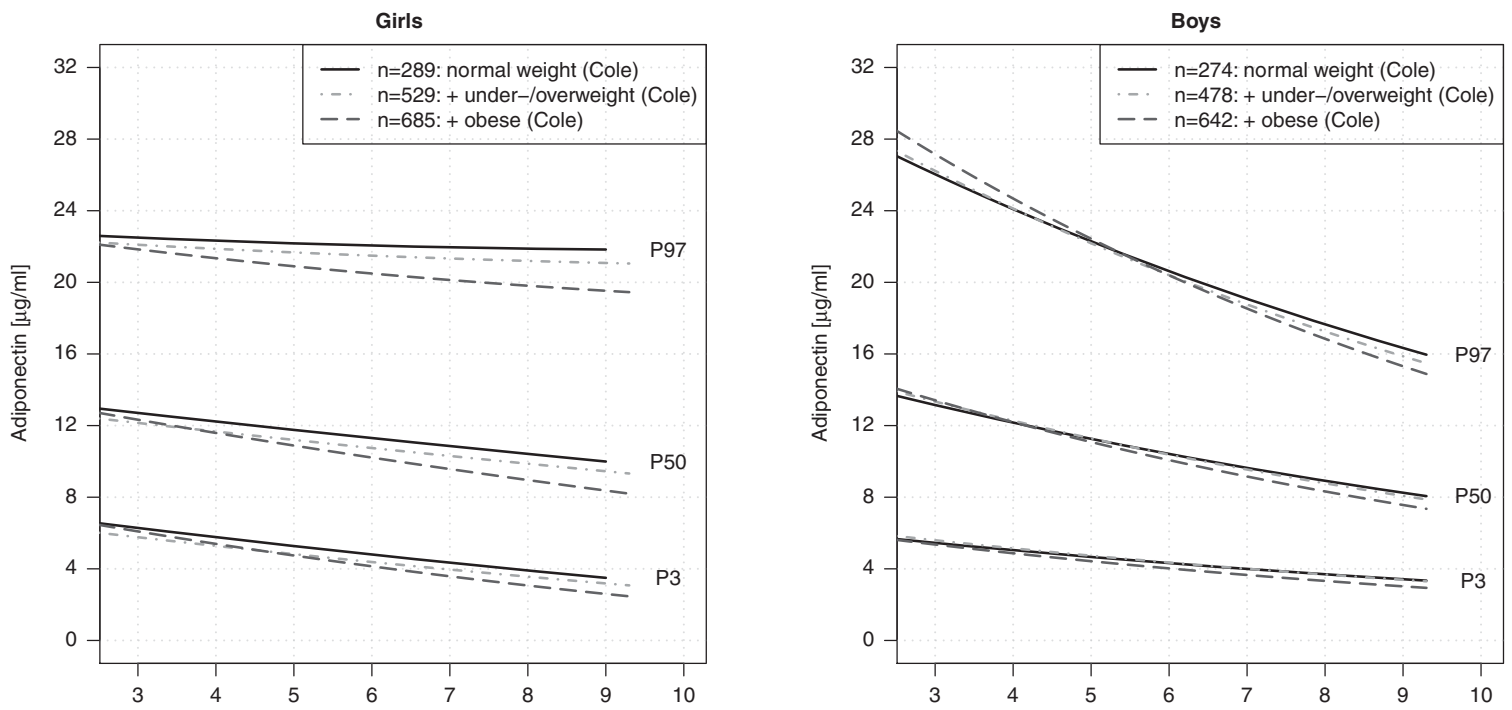

Figure 4. Smoothed percentile curves (3rd, 50th and 97th percentile) for adiponectin $\left(\mu \mathrm{g} \mathrm{ml}^{-1}\right)$ according to BMI categories among children aged 2.0-9.9 years modelled by GAMLSS. 

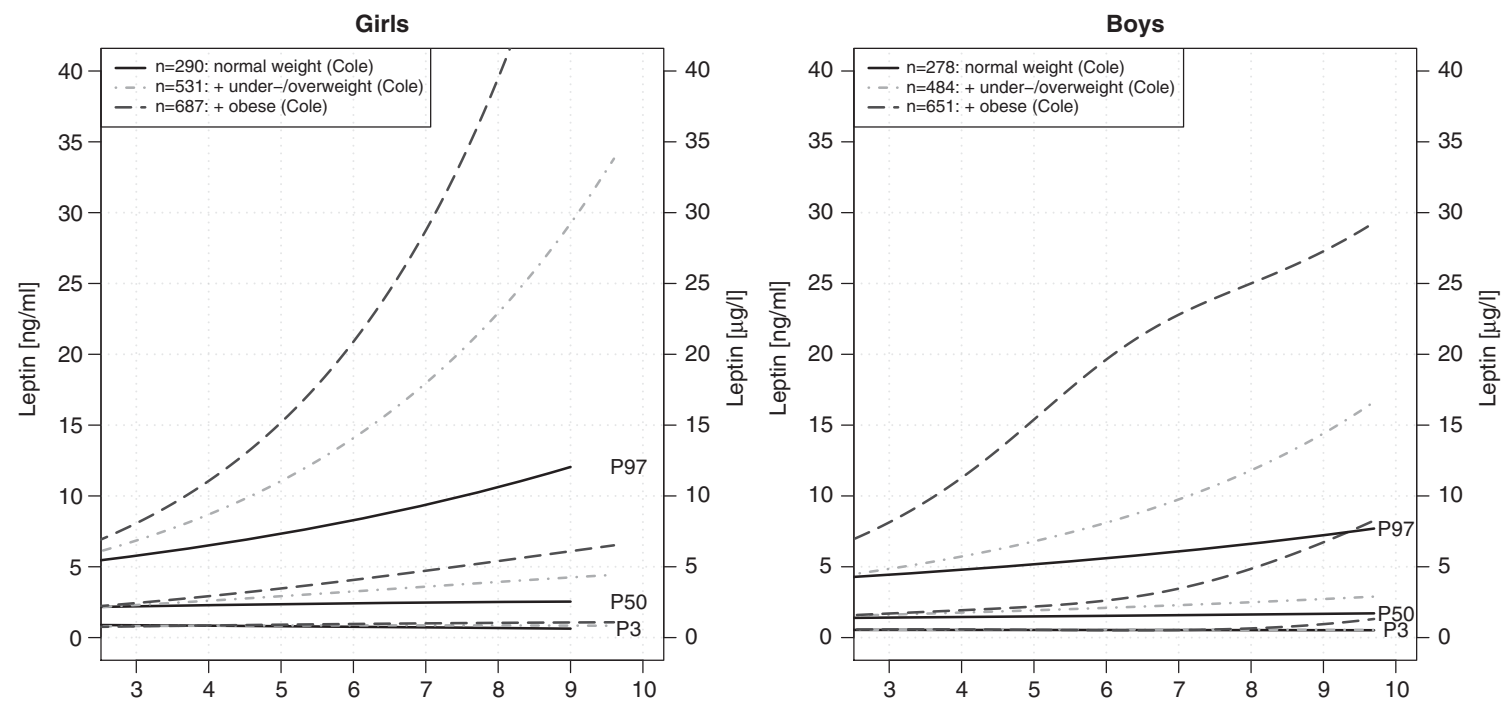

Figure 5. Smoothed percentile curves (3rd, 50th and 97th percentile) for leptin ( $\mathrm{ng} \mathrm{ml}^{-1}$ ) according to BMI categories among children aged 2.0-9.9 years modelled by GAMLSS.

789 normal-weight children found that the leptin levels rose from 5 to 15 years of age in parallel with total body weight.

In summary, according to our knowledge, the presented ageand sex-specific percentiles of leptin and adiponectin are the first ones based on a European multinational non-clinical children's population, which is the most important strength of our study. In addition, the applied statistical modelling was based on most recent, advanced methodology.

There might be possible selection effects due to the construction of the obesity case-control study. Not all countries are included in the case-control study; Cyprus had to be excluded because they did not collect venous blood, and other countries, for example, Italy (30\%), Germany (21\%) and Hungary (20\%) are overrepresented and Belgium (1\%) and Sweden (7\%) are underrepresented, remaining Estonia (11\%) and Spain (10\%). Despite the fact that Southern Europe is somewhat overrepresented and Western Europe is underrepresented, we have a good coverage of Central, Northern and Eastern Europe compared with the proportion of population living in these areas. Furthermore, our analysis group does not differ from the IDEFICS baseline population with respect to the age of parents, birth weight and months of breastfeeding. In addition, our analysis group also shows the same age distribution and only a slightly different sex distribution (53\% girls in our analysis group compared with $49 \%$ in the study population). But families of children in the analysis group show on average a lower income level (71\% have an ISCED level of 4 or lower versus $60 \%$ in the IDEFICS baseline survey) and lower level of education (70\% have a medium or lower income level versus $64 \%$ in the IDEFICS baseline survey). The main limitation of our results is the relatively small number of normalweight children. We applied different strategies as weighted GAMLSS regression and bootstrap strategies to compensate for the selection effect of obese and overweight children in the nested case-control study. However, none of the approaches could diminish the strong association of BMI on hormone levels, especially on leptin. Therefore, we restricted the analysis to normal-weight children only. The small sample size might have an influence on the reliability of the curves. For this reason, these findings should be used with care in clinical practise and should rather be used as support for diagnosis than as a decision tool.

\section{CONCLUSION}

The reference values presented for leptin and adiponectin in mostly prepubertal, normal-weight children are the first ones that are based on a multinational non-clinical children cohort study. These percentile values and curves have have an important role in clinical practise and in epidemiological studies, because these hormones are frequently measured in centres dealing with endocrinology, diabetes and obesity, but the interpretation of the individual values is hampered by the lack of valid reference values. We hope that the percentiles presented here will help to overcome this problem.

\section{CONFLICT OF INTEREST}

The authors declare no conflict of interest.

\section{ACKNOWLEDGEMENTS}

This work was done as part of the IDEFICS Study (www.idefics.eu). We gratefully acknowledge the financial support of the European Community within the Sixth RTD Framework Programme Contract No. 016181 (FOOD). We are grateful to the Volkswagen Foundation that financially supported the production of this supplement.

\section{DISCLAIMER}

The information in this document reflects the author's view and is provided as is.

\section{REFERENCES}

1 Mattu HS, Randeva HS. Role of adipokines in cardiovascular disease. J Endocrinol 2013; 216: T17-T36.

2 Mantzoros CS. The role of leptin in human obesity and disease: a review of current evidence. Ann Intern Med 1999; 130: 671-680.

3 Raucci R, Rusolo F, Sharma A, Colonna G, Castello G, Costantini S. Functional and structural features of adipokine family. Cytokine 2013; 61: 1-14.

4 Matsubara M, Maruoka S, Katayose S. Inverse relationship between plasma adiponectin and leptin concentrations in normal-weight and obese women. Eur J Endocrinol 2002; 147: 173-180.

5 Martos-Moreno GÁ, Barrios V, Argente J. Normative data for adiponectin, resistin, interleukin 6, and leptin/receptor ratio in a healthy Spanish pediatric population: relationship with sex steroids. Eur J Endocrinol 2006; 155: 429-434.

6 Al-Suhaimi EA, Shehzad A. Leptin, resistin and visfatin: the missing link between endocrine metabolic disorders and immunity. Eur J Med Res 2013; 18: 12. 
7 Böttner A, Kratzsch J, Müller G, Kapellen TM, Blüher S, Keller E et al. Gender differences of adiponectin levels develop during the progression of puberty and are related to serum androgen levels. J Clin Endocrinol Metab 2004; 89: 4053-4061.

8 Papaioannou HI, Stakos DA, Tziakas DN, Chalikias G, Tsigalou C, Kartali S et al. Plasma leptin and adiponectin concentrations in healthy, non-obese children. J Pediatr Endocrinol Metab 2011; 24: 313-318.

9 Kavazarakis E, Moustaki M, Gourgiotis D, Drakatos A, Bossios A, Zeis PM et al. Relation of serum leptin levels to lipid profile in healthy children. Metabolism 2001; 50: 1091-1094.

10 l'Allemand D, Schmidt S, Rousson V, Brabant G, Gasser T, Grüters A Associations between body mass, leptin, IGF-I and circulating adrenal androgens in children with obesity and premature adrenarche. Eur J Endocrinol 2002; 146 537-543.

11 Venner AA, Doyle-Baker PK, Lyon ME, Fung TS. A meta-analysis of leptin reference ranges in the healthy paediatric prepubertal population. Ann Clin Biochem 2009; 46: $65-72$.

12 Ahrens W, Bammann K, Siani A, Buchecker K, De Henauw S, lacoviello L et al. IDEFICS Consortium. The IDEFICS cohort: design, characteristics and participation in the baseline survey. Int J Obes (Lond) 2011; 35(Suppl 1): S3-S15.

13 Cole TJ, Bellizzi MC, Flegal KM, Dietz WH. Establishing a standard definition for child overweight and obesity worldwide: international survey. BMJ 2000; 320 1240-1243.

14 Cole T, Lobstein T. Extended international (IOTF) body mass index cut-offs for thinness, overweight and obesity. Pediatric Obesity 2012; 7: 284-294.

15 Rigby RA, Stasinopoulos DM. Generalized additive models for location, scale and shape (with discussion). Appl Stat 2005; 54: 507-554.

16 Cole T, Stanojevic S, Stocks J, Coates A, Hankinson J, Wade A. Age-and size-related reference ranges: a case study of spirometry through childhood and adulthood Stat Med 2009; 28: 880-898.
17 Stasinopoulos DM, Rigby RA. Generalized additive models for location scale and shape (GAMLSS) in R. J Stat Softw 2007; 23: 1-46.

18 van Buuren S, Fredriks M. Worm plot: a simple diagnostic device for modelling growth reference curves. Stat Med 2001; 20: 1259-1277.

19 Wilasco MIA, Goldani HA, Dornelles CT, Maurer RL, Kieling CO, Porowski M et al Ghrelin, leptin and insulin in healthy children: Relationship with anthropometry, gender, and age distribution. Regul Pept 2012; 173: 21-26.

20 Murphy MJ, Hosking J, Metcalf BS, Voss LD, Jeffery AN, Sattar N et al. Distribution of adiponectin, leptin, and metabolic correlates of insulin resistance: a longitudinal study in British children; 1: prepuberty (EarlyBird 15). Clin Chem 2008; 54: 1298-1306.

21 Lundblad RL. Considerations for the use of blood plasma and serum for proteomic analysis. Internet J Genome Proteo 2005; 1.

22 Garcia-Mayor RV, Andrade MA, Rios M, Lage M, Dieguez C, Casanueva FF. Serum leptin levels in normal children: relationship to age, gender, body mass index pituitary-gonadal hormones, and pubertal stage. J Clin Endocrinol Metab 1997; 82: 2849-2855.

23 Nagy $P$, Kovacs E, Moreno LA, Veidebaum T, Tornaritis $M$, Kourides $Y$ et al. Percentile reference values for anthropometric body composition indices in European children from the IDEFICS study. Int J Obes (Lond) 2014; 38(Suppl 2); S15-S25.

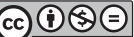

This work is licensed under a Creative Commons AttributionNonCommercial-NoDerivs 4.0 International License. The images or other third party material in this article are included in the article's Creative Commons license, unless indicated otherwise in the credit line; if the material is not included under the Creative Commons license, users will need to obtain permission from the license holder to reproduce the material. To view a copy of this license, visit http:// creativecommons.org/licenses/by-nc-nd/4.0/ 